\title{
AN ALGEBRA OF SEQUENCES OF FUNCTIONS, WITH AN APPLICATION TO THE BERNOULLIAN FUNCTIONS*
}

BY

E. T. BELL

On account of its simplicity, its immediate applicability to many functions already in existence, and its suggestiveness with regard to generalizations of known functions or the creation of new, the algebra in question merits the somewhat detailed exposition in Part I. As its use is perhaps best seen from an example, we have sketched briefly in Part II the outlines of a new theory of the relations between the Bernoullian and Eulerian functions of any arguments and any ranks, showing that by means of the algebra all necessary computations are reduced to a minimum. These functions are of two variables, of which one is complex and the other, the rank, a positive integer. The algebra establishes a simple isomorphism between the theory of relations between the functions and the like for the ordinary sine and cosine. (It is necessary here to distinguish between ordinary and umbral circular functions; the nature of the distinction appears presently.) In a paper which I expect to publish later there is a more detailed application of this algebra to certain new functions, suggested by the algebra, of three variables, of which two are complex and the third a positive integer. For unit values of one of the complex variables these functions degenerate to the Bernoullian and Eulerian functions of Part II; for unit values of the other, they become certain polynomials, of considerable importance in the arithmetical theory of quadratic forms, discussed on several occasions by Hermite, Weierstrass and, more recently, by Bulyguin and Gruder. In this application there are simple isomorphisms with the circular, the hyperbolic and the elliptic functions. In a third paper, to appear shortly, I have shown how the algebra gives at once the complete theory of the relations between the functions of Spitzer, which include as special cases the Bessel coefficients. Here there is simple isomorphism with the exponential function. By a generalization of the exponential function, $\sum_{m} x^{m+\nu} / \Gamma(m+\nu)$, in which $x, \nu$ are complex, I have shown in a paper not yet published that the algebra is readily applicable to the Bessel functions. The algebra therefore is of considerable utility. Its chief use, how-

\footnotetext{
- Presented to the Society, September 11, 1925; received by the editors in April, 1925.
} 
ever, is its suggestiveness as to new functions to be investigated, and its immediate forecasts of the results to be expected. For example, in a paper to be published elsewhere, I have shown that the ultra-Bernoullian functions of Krause and Appell (functions of two complex variables) find their ready generalizations and complete theory by a simple inspection of the symbolic formulas in Part I, and that the number of distinct generalizations possible is infinite. Of rather more importance is the similar generalization of the functions of Olivier, also suggested by the algebra, as these generalizations must appear sooner or later in the study of any sequences of functions (cf. \$13).

The umbral calculus of Blissard is used freely in the sequel. Brief but sufficient synopses of the main processes of this powerful but neglected instrument being readily available in the places cited,* we shall assume them known. We presuppose also, $\$ 7$, end, the legitimacy of operating with infinite series in the manner established in a former paper, $\dagger$ but this assumption affects nothing in Part II or in any previous results in Part I. If preferred all reference to infinite series may be obviated, and operations with series may be replaced, as recently shown by Wedderburn, $\ddagger$ by others in a certain algebra with an infinite basis. Or again, the theorems, when found by either of these methods, can be proved independently if wished by an easy application of mathematical induction.

Part II is not intended in any way as a systematic treatment of the functions discussed, but merely as a collection of examples illustrating some of the results of Part I. Anyone skimming the paper is advised to ascertain from the context what letters denote umbrae; otherwise the point of most of the formulas will be missed.

\section{Part I. The Symmetric Functions $\varphi, \psi, \chi$}

1. A sequence of functions implies in the definition of a particular general element of the sequence the presence of at least one positive or negative integer, and conversely if in the definition of the general element of a set of functions there occurs at least one integer, the functions of the set can be ordered with respect to this integer into a sequence. If the integer is negative a suitable change in the definition or notation of the functions replaces it by a positive integer. The positive integer is called

* (1) Umbral symmetric functions ..., Ma the ma tische Zeitschrift, vol. 19 (1923), pp. $35-6, \$ \$ 1,2$; (2) $A$ revision of the Bernoullian functions, Bulletin of the American Mathematical Society, vol. 28 (1922), pp. 443-44, \$1.

$\dagger$ Euler algebra, these Tra n sa c tion s, vol. 25 (1923), pp. 135-54.

$\ddagger$ These Transactions, vol. 26 (1924), p. 421, formula (9.21). 
the rank. Hence the ranks in any sequence of functions can be taken as $0,1,2, \cdots$.

With respect to a fixed positive integer $m$ as modulus the ranks of the functions can be segregated into $m$ classes. The functions of the $r$ th class, $0 \leqq r<m$, are those whose ranks are $k m+r(k=0,1,2, \cdots)$.

The algebra which we are about to construct is fixed by the integer $m$, and for a definite $m$ is said to be of order $m$. This is not the usual definition of the order of an algebra, but is closely analogous to it. Instead of $m$ basal units of an algebra we have here $m+1$ types of symmetric functions, as will appear presently. When $m=2$, the case with which we shall be concerned here, the algebra is simply isomorphic to the theory of either the circular or the hyperbolic functions; when $m=1$ the isomorphism is with the function exp $a x, a \equiv$ an umbra. Now it is well known that the entire theory of the circular (or hyperbolic) functions is implied by (1) the theorem that $x^{2}+y^{2}$, where $x$ is real and $y$ real (or pure imaginary), reproduces itself with respect to multiplication, and (2) the integer solutions of $x^{2}+y^{2}=1$. A corresponding pair of theorems unifies the algebras for all orders $m \geqq$ ? : (1) the norm of any algebraic integer is self-reproductive under multiplication, (2) the units in the associated field of degree $m$, when for this (algebraic number) field is taken that defined by a primitive $m$ th root of either 1 or -1 . The corresponding functions for $m>2$ are in the simplest instance those of Olivier, in the general case the umbral generalization of these which is indicated in $\$ 13$. This is not further developed here, and is mentioned merely to show the simple, common basis underlying the algebras of all orders $>1$. The connection with sequences of the following sections is made in $\$ 8$.

2. Let each of $e_{j}, e_{j}^{\prime}(j=1,2, \cdots)$ denote a definite one of $1,-1$. Let first $u_{j}, v_{j}(j=1,2, \cdots)$ be ordinaries (elements of any field), and define $\varphi, \psi, \chi$ by

$$
\varphi_{2 n}(u)=u^{2 n}, \quad \psi_{2 n+1}(v)=v^{2 n+1},
$$

(2) $\psi_{n}\left(v_{1}, v_{2}, \ldots \cdot, v_{8}\right) \equiv \Sigma e_{2}^{\prime} e_{3}^{\prime} \cdot \cdots e_{8}^{\prime}\left(v_{1}+e_{2}^{\prime} v_{2}+e_{3}^{\prime} v_{3}+\cdots \cdot+e_{8}^{\prime} v_{8}\right)^{n}$,

(3) $\chi_{n}\left(u_{1}, u_{2}, \cdot . \cdot, u_{r} ; v_{1}, v_{2}, \cdot \cdot, v_{8}\right) \equiv \Sigma e_{1}{ }^{\prime} e_{2}^{\prime} \cdot . \cdot e_{8}^{\prime}\left(u_{1}+e_{2} u_{2}+\right.$

$$
\text { -. + } \left.+e_{r} u_{r}+e_{1}^{\prime} v_{1}+e_{2}^{\prime} v_{2}+\cdots+e_{\varepsilon}^{\prime} v_{\varepsilon}\right)^{n} \text {, }
$$

where $n \geqq 0$ is an integer, and the summations refer to all possible values of the $e_{j}, e_{k}{ }^{\prime}$. To simplify the writing, put henceforth

$$
u_{1}, u_{2}, \cdots \cdot, u_{r} \equiv U_{r} \equiv U, v_{1}, v_{2}, \cdots, v_{8} \equiv V_{c} \equiv V
$$


and similarly for accented letters, $u_{1}{ }^{\prime}, u_{2}{ }^{\prime}, \cdots, u_{p}{ }^{\prime} \equiv U_{p}{ }^{\prime}$, etc., the $U, V$ without suffixes being used when it is unnecessary to distinguish between even and odd values of $r, s$. Each of $u, u^{\prime}, u^{\prime \prime}, \ldots, v, v^{\prime}, v^{\prime \prime}, \ldots$ (without a suffix) denotes a single ordinary or a single umbra, according to the context in which it occurs. The functions (1), (2), (3) differ from those denoted in previous papers by the same letters. For functions of precisely two arguments we have the useful formulas

$$
\chi_{n}(u ; v)=\psi_{n}(u, v), \quad \chi_{2 n+1}(u ; v)=\psi_{2 n+1}(v, u) .
$$

From (1) - (3) we write down the following generators, in which the sines and cosines on the right are umbral:

$$
\begin{gathered}
2^{r-1} \prod_{j=1}^{r} \cos u_{j} x=\cos \varphi\left(U_{r}\right) x, \\
2^{2 s-1}(-1)^{s} \prod_{j=1}^{2 s} \sin v_{j} x=\cos \psi\left(V_{2 s}\right) x, \\
2^{2 s}(-1)^{2 s+1} \sum_{j=1}^{2 s+1} \sin v_{j} x=\sin \psi\left(V_{2 s+1}\right) x, \\
2^{r+2 s-1}(-1)^{s} \prod_{j=1}^{r} \cos u_{j} x \cdot \prod_{k=1}^{2 s} \sin v_{k} x=\cos \chi\left(U_{r} ; V_{2 s}\right) x \\
2^{r+2 s}(-1)^{\bullet} \prod_{j=1}^{r} \cos u_{j} x \cdot \prod_{k=1}^{2 s+1} \sin v_{k} x=\sin \chi\left(U_{r} ; V_{2 s+1}\right) x .
\end{gathered}
$$

It follows that $\varphi_{2 n}\left(U_{r}\right)$ is symmetric in $u_{1}, u_{2}, \cdots, u_{r} ; \psi_{n}\left(V_{s}\right)$ is symmetric in $v_{1}, v_{2}, \cdots, v_{s} ; \chi_{n}\left(U_{r} ; V_{s}\right)$ is symmetric in $u_{1}, u_{2}, \cdots, u_{r}$ and in $v_{1}, v_{2}, \cdots, v_{s}$. Notice that in (5) - (9) only those $\psi_{n}\left(V_{s}\right), \chi_{n}\left(U_{r} ; V_{s}\right)$ occur in which $n, s$ are of like parity, also that only $\varphi$ 's of even rank appear. This holds in all subsequent formulas.

Next let the $u_{j}, v_{k}$ be umbrae. Then by the elements of the umbral calculus as developed in the papers cited, it follows that (5)-(9) are valid for the new interpretations of (1)-(3). This remark is necessary for the reading of the formulas in the next section and thereafter. We recall that any umbral formula implies as a special case the same formula when all letters therein are interpreted to be ordinaries.

3. The $\varphi, \psi, \chi$ have three kinds of addition theorems, the first of which expresses a function of $m+n$ arguments $(m, n>0)$ as a bilinear form in functions of $m^{\prime}$ and $n^{\prime}$ arguments, where $m^{\prime}+n^{\prime}=m+n$. These theorems are found by replacing in (5)-(9) the $u_{i}, v_{k}$ by new arguments $u_{i}^{\prime}, v_{k}^{\prime}$, and $(r, s)$ by $(p, t)(r, s, p, t>0)$, multiplying member by member the 
identities thus obtained by the old, reducing the products by inspection of (5)-(9) to the same forms as these, and using (4) when necessary to replace on the right $\chi$ by $\psi$. Comparing coefficients of like powers of $x$ in the results we get in this way the following set of addition theorems of the first kind :

$$
\begin{aligned}
& \varphi_{2 n}\left(U_{r}, U_{p}^{\prime}\right)=\varphi_{2 n}\left(\varphi\left(U_{r}\right), \varphi\left(U_{p}^{\prime}\right)\right), \\
& \chi_{2 n}\left(U_{r}, U_{p}^{\prime} ; V_{2 s}, V_{2 t}^{\prime}\right)=\varphi_{2 n}\left(\chi\left(U_{r} ; V_{2 s}\right), \chi\left(U_{p}^{\prime} ; V_{2 t}^{\prime}\right)\right), \\
& \chi_{2 n}\left(U_{r}, U_{p}^{\prime} ; V_{2 s+1}, V_{2 t+1}^{\prime}\right)=\psi_{2 n}\left(\chi\left(U_{r} ; V_{2 s+1}\right), \chi\left(U_{p}^{\prime} ; V_{2 t+1}^{\prime}\right)\right), \\
& \chi_{2 n}\left(U_{r}, U_{p}^{\prime} ; V_{2 s}\right)=\varphi_{2 n}\left(\varphi\left(U_{r}\right), \chi\left(U_{p}^{\prime} ; V_{2 s}\right)\right), \\
& \chi_{2 n+1}\left(U_{r}, U_{p}^{\prime} ; V_{2 s+1}\right)=\psi_{2 n+1}\left(\varphi\left(U_{r}\right), \chi\left(U_{p}^{\prime} ; V_{2 s+1}\right)\right), \\
& \chi_{2 n+1}\left(U_{r}, U_{p}^{\prime} ; V_{2 s}, V_{2 t+1}^{\prime}\right)=\psi_{2 n+1}\left(\chi\left(U_{r} ; V_{2 s}\right), \chi\left(U_{p}{ }^{\prime} ; V_{2 t+1}\right)\right) .
\end{aligned}
$$

It is convenient to have explicitly the special forms obtained upon suppressing one or both of $U_{r}, U_{p}^{\prime}$ and applying

$$
\chi_{2 n}\left(0 ; V_{2 s}\right)=\psi_{2 n}\left(V_{2 s}\right), \chi_{2 n+1}\left(0 ; V_{2 s+1}\right)=\psi_{2 n+1}\left(V_{2 s+1}\right)
$$

to the results. We find

$$
\begin{gathered}
\psi_{2 n}\left(V_{2 s}, V_{2 t}^{\prime}\right)=\varphi_{2 n}\left(\varphi\left(V_{2 s}\right), \psi\left(V_{2 t}^{\prime}\right)\right), \\
\psi_{2 n}\left(V_{2 s+1}, V_{2 t+1}^{\prime}\right)=\psi_{2 n}\left(\psi\left(V_{2 s+1}\right), \psi\left(V_{2 t+1}^{\prime}\right)\right), \\
\psi_{2 n+1}\left(V_{2 s}, V_{2 t+1}^{\prime}\right)=\psi_{2 n+1}\left(\psi\left(V_{2 s}\right), \psi\left(V_{2 t+1}^{\prime}\right)\right) ;
\end{gathered}
$$

and for the $\chi$ functions of even rank,

$$
\begin{gathered}
\chi_{2 n}\left(U_{r} ; V_{2 s}\right)=\varphi_{2 n}\left(\varphi\left(U_{r}\right), \psi\left(V_{2 s}\right)\right), \\
\chi_{2 n}\left(U_{r} ; V_{2 s}, V_{2 t}^{\prime}\right)=\varphi_{2 n}\left(\psi\left(V_{2 s}\right), \chi\left(U_{r} ; V_{2 t}^{\prime}\right)\right), \\
\chi_{2 n}\left(U_{r} ; V_{2 s+1}, V_{2 t+1}^{\prime}\right)=\psi_{2 n}\left(\psi\left(V_{2 s+1}\right), \chi\left(U_{r} ; V_{2 t+1}^{\prime}\right)\right) ;
\end{gathered}
$$

while for the odd ranks,

$$
\begin{gathered}
\chi_{2 n+1}\left(U_{r} ; V_{2 s+1}\right)=\psi_{2 n+1}\left(\varphi\left(U_{r}\right), \psi\left(V_{2 s+1}\right)\right), \\
\chi_{2 n+1}\left(U_{r} ; V_{2 s}, V_{2 t+1}^{\prime}\right)=\psi_{2 n+1}\left(\psi\left(V_{2 s}\right), \chi\left(U_{r} ; V_{2 t+1}^{\prime}\right)\right), \\
\chi_{2 n+1}\left(U_{r} ; V_{2 s+1}, V_{2 t}^{\prime}\right)=\psi_{2 n+1}\left(\psi\left(V_{2 s+1}\right), \chi\left(U_{r} ; V_{2 t}^{\prime}\right)\right) .
\end{gathered}
$$


Suppression of $U_{r}$ or $U_{p}^{\prime}$ is equivalent to replacing each $u_{i}$ or each $u_{j}^{\prime}$ by zero. We cannot similarly delete either $V_{0}$ or $V_{t}^{\prime}$, for this would be equivalent to taking $v_{k}=0$ or $v_{k}{ }^{\prime}=0(k=1,2, \cdots)$ in (6)-(9), which obviously is not permissible.

4. The second type of addition theorem expresses the functions of arguments $u_{i}+u_{i}{ }^{\prime}$ linearly in terms of functions of arguments $u_{i}$ and $u_{i}^{\prime}(j=$ $1,2, \cdots)$ respectively. They are found by replacing any $u_{i}$ or $v_{k}$ in (5)-(9) by the sum of two others, expanding the left of the identities so obtained by the addition theorems for the sine and cosine, and using (5)-(9) to reduce the results to umbral sines or cosines of new functions $\varphi, \psi, \chi$. We find (with $U \equiv U_{r}, V \equiv V_{s}$ as explained in $\S 2$ ),

$$
\begin{aligned}
2 \varphi_{2 n}\left(U, u^{\prime}+u^{\prime \prime}\right) & =\varphi_{2 n}\left(U, u^{\prime}, u^{\prime \prime}\right)-\chi_{2 n}\left(U ; u^{\prime}, u^{\prime \prime}\right), \\
2 \psi_{n}\left(V, v^{\prime}+v^{\prime \prime}\right) & =\chi_{n}\left(v^{\prime} ; V, v^{\prime \prime}\right)+\chi_{n}\left(v^{\prime \prime} ; V, v^{\prime}\right), \\
2 \chi_{n}\left(U, u^{\prime}+u^{\prime \prime} ; V\right) & =\chi_{n}\left(U, u^{\prime}, u^{\prime \prime} ; V\right)-\chi_{n}\left(U ; V, u^{\prime}, u^{\prime \prime}\right), \\
2 \chi_{n}\left(U ; V, v^{\prime}+v^{\prime \prime}\right) & =\chi_{n}\left(U, v^{\prime} ; V, v^{\prime \prime}\right)+\chi_{n}\left(U, v^{\prime \prime} ; V, v^{\prime}\right) .
\end{aligned}
$$

Applying either of (19), (20) to the other we get

$$
\begin{aligned}
& 4 \chi_{n}\left(U, u^{\prime}+u^{\prime \prime} ; V, v^{\prime}+v^{\prime \prime}\right) \\
& =\chi_{n}\left(U, u^{\prime}, u^{\prime \prime}, v^{\prime} ; V, v^{\prime \prime}\right)+\chi_{n}\left(U, u^{\prime}, u^{\prime \prime}, v^{\prime \prime} ; V, v^{\prime}\right) \\
& \quad-\chi_{n}\left(U, v^{\prime} ; V, u^{\prime}, u^{\prime \prime}, v^{\prime \prime}\right)-\chi_{n}\left(U, v^{\prime \prime} ; V, u^{\prime}, u^{\prime \prime}, v^{\prime}\right) .
\end{aligned}
$$

5. There are two types of subtraction theorems. The second is indicated in $\S 6$; the first is found similarly to the preceding addition theorems, or directly from them by means of (29), which is obvious independently. We have

$$
\begin{gathered}
2 \varphi_{2 n}\left(U, u^{\prime}-u^{\prime \prime}\right)=\varphi_{2 n}\left(U, u^{\prime}, u^{\prime \prime}\right)+\chi_{2 n}\left(U ; u^{\prime}, u^{\prime \prime}\right), \\
2 \psi_{n}\left(V, v^{\prime}-v^{\prime \prime}\right)=-\chi_{n}\left(v^{\prime} ; V, v^{\prime \prime}\right)+\chi_{n}\left(v^{\prime \prime} ; V, v^{\prime}\right), \\
2 \chi_{n}\left(U, u^{\prime}-u^{\prime \prime} ; V\right)=\chi_{n}\left(U, u^{\prime}, u^{\prime \prime} ; V\right)+\chi_{n}\left(U ; V, u^{\prime}, u^{\prime \prime}\right), \\
2 \chi_{n}\left(U ; V, v^{\prime}-v^{\prime \prime}\right)=-\chi_{n}\left(U, v^{\prime} ; V, v^{\prime \prime}\right)+\chi_{n}\left(U, v^{\prime \prime} ; V, v^{\prime}\right),
\end{gathered}
$$

and hence, from the last two,

$$
\begin{aligned}
& 4 \chi_{n}\left(U, u^{\prime}-u^{\prime \prime} ; V, v^{\prime}-v^{\prime \prime}\right) \\
& =-\chi_{n}\left(U, u^{\prime}, u^{\prime \prime}, v^{\prime} ; V, v^{\prime \prime}\right)+\chi_{n}\left(U, u^{\prime} u^{\prime \prime}, v^{\prime \prime} ; V, v^{\prime}\right) \\
& \quad-\chi_{n}\left(U, v^{\prime} ; V, u^{\prime}, u^{\prime \prime}, v^{\prime \prime}\right)+\chi_{n}\left(U, v^{\prime \prime} ; V, u^{\prime}, u^{\prime \prime}, v^{\prime}\right) .
\end{aligned}
$$


Similarly from (19), (25) we get

$$
\begin{aligned}
4 \chi_{n}\left(U, u^{\prime}+u^{\prime \prime} ; V, v^{\prime}-v^{\prime \prime}\right) & \\
= & -\chi_{n}\left(U, u^{\prime}, u^{\prime \prime}, v^{\prime} ; V, v^{\prime \prime}\right)+\chi_{n}\left(U, u^{\prime}, u^{\prime \prime}, v^{\prime \prime} ; V, v^{\prime}\right) \\
& +\chi_{n}\left(U, v^{\prime} ; V, u^{\prime}, u^{\prime \prime}, v^{\prime \prime}\right)-\chi_{n}\left(U, v^{\prime \prime} ; V, u^{\prime}, u^{\prime \prime}, v^{\prime}\right),
\end{aligned}
$$

and from (20), (24),

$$
\begin{aligned}
4 \chi_{n}(U, & \left.u^{\prime}-u^{\prime \prime} ; V, v^{\prime}+v^{\prime \prime}\right) \\
= & \chi_{n}\left(U, u^{\prime}, u^{\prime \prime}, v^{\prime} ; V, v^{\prime \prime}\right)+\chi_{n}\left(U, u^{\prime}, u^{\prime \prime}, v^{\prime \prime} ; V, v^{\prime}\right) \\
& +\chi_{n}\left(U, v^{\prime} ; V, u^{\prime}, u^{\prime \prime}, v^{\prime \prime}\right)+\chi_{n}\left(U, v^{\prime \prime} ; V, u^{\prime}, u^{\prime \prime}, v^{\prime}\right) .
\end{aligned}
$$

Combine (17)-(25) and find

$$
\begin{aligned}
\psi_{n}(V,-v) & =-\psi_{n}(V, v), \chi_{n}(U ; V,-v)=-\chi_{n}(U ; V, v), \\
\varphi_{2 n}\left(U, u^{\prime}, u^{\prime \prime}\right) & =\varphi_{2 n}\left(U, u^{\prime}+u^{\prime \prime}\right)+\varphi_{2 n}\left(U, u^{\prime}-u^{\prime \prime}\right), \\
\chi_{2 n}\left(U ; v^{\prime}, v^{\prime \prime}\right) & =\varphi_{2 n}\left(U, v^{\prime}-v^{\prime \prime}\right)-\varphi_{2 n}\left(U, v^{\prime}+v^{\prime \prime}\right), \\
\chi_{n}(u ; V, v) & =\psi_{n}(V, u+v)-\psi_{n}(V, u-v), \\
\chi_{n}\left(U, u^{\prime}, u^{\prime \prime} ; V\right) & =\chi_{n}\left(U, u^{\prime}+u^{\prime \prime} ; V\right)+\chi_{n}\left(U, u^{\prime}-u^{\prime \prime} ; V\right), \\
\chi_{n}\left(U ; V, v^{\prime}, v^{\prime \prime}\right) & =\chi_{n}\left(U, v^{\prime}-v^{\prime \prime} ; V\right)-\chi_{n}\left(U, v^{\prime}+v^{\prime \prime} ; V\right), \\
\chi_{n}(U, u ; V, v) & =\chi_{n}(U ; V, u+v)-\chi_{n}(U ; V, u-v),
\end{aligned}
$$

and hence from (29), (32), (35)

$$
\begin{gathered}
\chi_{n}(u ; V, v)=\psi_{n}(V, v+u)+\psi_{n}(V, v-u), \\
\chi_{n}(U, u ; V, v)=\chi_{n}(U ; V, v+u)+\chi_{n}(U ; V, v-u) .
\end{gathered}
$$

To condense the similar formulas obtained from (21), (26)-(28), denote the arguments of the $\chi_{n}$ occurring in the left of these identities by $A, B, C, D$ respectively, and those in the right of (26), in the order in which they are written, by $a, b, c, d$ respectively. Then

$$
\begin{aligned}
& \chi_{n}(a)=\chi_{n}(A)-\chi_{n}(B)-\chi_{n}(C)+\chi_{n}(D), \\
& \chi_{n}(b)=\chi_{n}(A)+\chi_{n}(B)+\chi_{n}(C)+\chi_{n}(D), \\
& \chi_{n}(c)=-\chi_{n}(A)-\chi_{n}(B)+\chi_{n}(C)+\chi_{n}(D), \\
& \chi_{n}(d)=-\chi_{n}(A)+\chi_{n}(B)-\chi_{n}(C)+\chi_{n}(D),
\end{aligned}
$$


giving the interesting formula

$$
\chi_{n}^{2}(a)+\chi_{n}^{2}(b)+\chi_{n}^{2}(c)+\chi_{n}^{2}(d)=4\left[\chi_{n}^{2}(A)+\chi_{n}^{2}(B)+\chi_{n}^{2}(C)+\chi_{n}^{2}(D)\right],
$$

which recalls the identity on which Jacobi founded the theory of elliptic functions. Some curious applications of this identity will be given in other papers; applied to the Bernoullian and Eulerian functions of Part II it yields a remarkable system of relations between squares of sums of these functions which will be developed in a separate note.

6. The addition theorems of the third kind are the resultants of those of the first two kinds, and are among the most important processes of this algebra in its applications. They are so numerous, however, that we shall not take space to write out the set, which can be found from $\$ \S 3-5$, but will merely indicate their nature and write down the principal transformations for obtaining them. Any systematic investigation of a set of sequences of functions presupposes the complete system of such theorems. To derive them we transform the right hand members of (17)-(37) by means of all the formulas in $\S 3$, recalling from $\S 2$ that $\varphi_{2 n}(u)=u^{2 n}, \psi_{2 n+1}(v)=v^{2 n+1}$. With the exception of those required for (21), (26)-(28), (38)-(42), the complete set of necessary transformation formulas is

$$
\begin{aligned}
\varphi_{2 n}\left(U, u^{\prime}, u^{\prime \prime}\right) & =\varphi_{2 n}\left(\varphi(U), \varphi\left(U^{\prime}, u^{\prime \prime}\right)\right)=\varphi_{2 n}\left(u^{\prime}, \varphi\left(U, u^{\prime \prime}\right)\right), \\
\chi_{2 n}\left(U ; v^{\prime}, v^{\prime \prime}\right) & =\varphi_{2 n}\left(\varphi(U), \psi\left(v^{\prime}, v^{\prime \prime}\right)\right)=\psi_{2 n}\left(v^{\prime}, \chi\left(U, v^{\prime \prime}\right)\right), \\
\lambda_{n}(u ; V, v) & =\psi_{n}(\psi(u, v), \psi(V))=\psi_{n}(v, \chi(u ; V)), \\
\chi_{2 n}\left(u ; V_{2 s+1}, v\right) & =\varphi_{2 n}\left(u, \psi\left(V_{2 s+1}, v\right)\right), \\
\chi_{2 n+1}\left(u ; V_{2 s}, v\right) & =\psi_{2 n+1}\left(u, \psi\left(V_{2 s}, v\right)\right) .
\end{aligned}
$$

For the excepted formulas there is a similar but longer list written down in the same way. An example of an addition theorem of the third kind is, by (17) and the above,

$$
2 \varphi_{2 n}\left(U, u^{\prime}+u^{\prime \prime}\right)=\varphi_{2 n}\left(u^{\prime}, \varphi\left(U, u^{\prime \prime}\right)\right)-\psi_{2 n}\left(u^{\prime}, \chi\left(U ; u^{\prime \prime}\right)\right)
$$

which by (4) has the special case

$$
2 \varphi_{2 n}\left(u, u^{\prime}+u^{\prime \prime}\right)=\varphi_{2 n}\left(u, \varphi\left(u, u^{\prime \prime}\right)\right)-\psi_{2 n}\left(u^{\prime}, \psi\left(u, u^{\prime \prime}\right)\right) .
$$

In the theory of Bernoullian functions and of many others the special cases of all the addition theorems in which each of $U \equiv U_{r}, V \equiv V_{\text {s }}$ contains not more than two umbrae are fundamental.

Clearly all of the results in $\$ \S 2-6$ can be verified without reference to (4)-(9). To do so, however, merely masks their straightforward origin and destroys much of their pliability in use. 
7. The processes of generalization outlined in this section unite, in the theory of any given set of sequences, numerous theorems into a single statement and at the same time give a wide extension to isolated results. By means of the umbral calculus any identity or equality between ordinaries can be immediately interpreted as the like between umbrae. The simple rules for performing and interpreting the umbral processes of a field, including umbral differentiation, were summarized in the papers cited in the introduction. We proceed to write out a few of the most frequently used umbral formulas obtainable in this way.

Let first $f(x)$ be a polynomial in the ordinary $x$, and $\lambda, \mu$ umbrae, $a, b$ ordinaries independent of $x$, and recall the definition of the rth umbral derivative of $\lambda_{n}$ with respect to $\lambda$ :

$$
D_{\lambda}^{r} \lambda^{n} \equiv D_{\lambda}^{r} \lambda_{n} \equiv \frac{n !}{(n-r) !} \lambda^{n-r} \equiv \frac{n !}{(n-r) !} \lambda_{n-r} .
$$

This coincides with the ordinary derivative when and only when $\lambda_{n}$ is its special case, the $n$th power of the ordinary $\lambda$. It is in general not a limiting process. We shall denote the $r$ th ordinary derivative of $f$ with respect to $x$ by the superscript $(r)$, thus $f^{(r)}$, using $f^{\prime}, f^{\prime \prime}$ when $r=1,2$. The sign of implication is as usual . . . .

Then evidently for $r, s$ constant positive integers and $n=0,1,2, \ldots$ we have the following implications:

$$
\begin{aligned}
a \lambda^{2 n s} & =b \mu^{2 n s} . \supset . a\left[f\left(x+\lambda^{s}\right)+f\left(x-\lambda^{s}\right)\right]=b\left[f\left(x+\mu^{s}\right)+f\left(x-\mu^{s}\right)\right], \\
a \lambda^{(2 n+1) s} & =b \mu^{(2 n+1) s} . \supset . a\left[f\left(x+\lambda^{s}\right)-f\left(x-\lambda^{s}\right)\right]=b\left[f\left(x+\mu^{s}\right)-f\left(x-\mu^{s}\right)\right], \\
a \lambda^{n s} & =b \mu^{n s} . \supset . a f\left(x+\lambda^{s}\right)=b f\left(x+\mu^{s}\right), \\
a \lambda^{n} & =b D_{\mu^{r}}^{\mu^{n}} . \supset . a f(x+\lambda)=b f^{(r)}(x+\mu) .
\end{aligned}
$$

The next also are easily seen, and each is of frequent occurrence :

$$
\begin{aligned}
& a x^{2 r} \sin \lambda x=b \sin \mu x . \supset . b \mu^{2 n+1}=a(-1)^{r} D_{\lambda}^{2 r} \lambda^{2 n+1} \\
& . \text {. . } a(-1)^{r}\left[f^{(2 r)}(x+\lambda)-f^{(2 r)}(x-\lambda)\right]=b[f(x+\mu)-f(x-\mu)] \\
& a x^{2 r+1} \sin \lambda x=b \cos \mu x . \supset .-b \mu^{2 n}=a(-1)^{r} D_{\lambda}^{2 r+1} \lambda^{2 n} \\
& . \text {. . } a(-1)^{r}\left[f^{(2 r+1)}(x+\lambda)-f^{(2 r+1)}(x-\lambda)\right]=-b[f(x+\mu)+f(x-\mu)] \\
& a x^{2 r} \cos \lambda x=b \cos \mu x . \supset . b \mu^{2 n}=a(-1)^{r} D_{\lambda}^{2 r} \lambda^{2 n} \\
& . \text {. . } a(-1)^{r}\left[f^{(2 r)}(x+\lambda)+f^{(2 r)}(x-\lambda)\right]=b[f(x+\mu)+f(x-\mu)]
\end{aligned}
$$




$$
\begin{aligned}
& a x^{2 r+1} \cos \lambda x=b \sin \mu x \cdot \supset . b \mu^{2 n+1}=a(-1)^{r} D_{\lambda}^{2 r+1} \lambda^{2 n+1}, \\
& . \supset . a(-1)^{r}\left[f^{(2 r+1)}(x+\lambda)+f^{(2 r+1)}(x-\lambda)\right]=b[f(x+\mu)-f(x-\mu)] ;
\end{aligned}
$$

$$
a \cos \lambda x=b \text { and } \sin \lambda x=0 . \supset . a f(x+\varphi(\lambda, 1))=b f(x) \text {. }
$$

It is convenient now to introduce a few definitions. For a particular general choice of the units $e_{2}, e_{3}, \cdots, e_{r}$ call $\left(e_{2}, e_{3}, \cdots, e_{r}\right)$ the signature of

$$
f\left(U_{p}+e_{2} u_{2}+e_{3} u_{3}+\cdots \cdot+e_{r} u_{r}\right) \text {, }
$$

in which $U_{p}$ involves no symbol $e, e_{j}, e_{k}{ }^{\prime}, e_{s}{ }^{\prime \prime}, \ldots$ of a unit. Denote by $\Sigma_{\sigma}$ a summation with respect to all possible values of the units occurring in the signature of the function following $\Sigma_{\sigma}$. If in the signature there are precisely $n$ units, $\boldsymbol{\Sigma}_{\sigma}$ consists of $2^{n}$ terms.

With $U_{r}, V_{s}$ as before ( $\$ 2$ ), we have now the important formulas, with $c=1$, or -1 ,

$$
\begin{array}{r}
f\left(x+c \varphi\left(U_{r}\right)\right)=\Sigma_{\sigma} f\left(x+c\left(u_{1}+e_{2} u_{2}+e_{3} u_{3}+\cdots+e_{r} u_{r}\right)\right), \\
f\left(x+c \psi\left(V_{s}\right)\right)=\Sigma_{\sigma} e_{2} e_{3} \cdot \cdot e_{s} f\left(x+c\left(v_{1}+e_{2} v_{2}+\cdots \cdot+e_{s} v_{s}\right)\right), \\
f\left(x+c \chi\left(U_{r} ; V_{s}^{\prime}\right)\right)=\Sigma_{\sigma} e_{1}^{\prime} e_{2}^{\prime} \cdot \cdot e_{s}^{\prime} f\left(x+c\left(u_{1}+e_{2} u_{2}+\cdots \cdot+e_{r} u_{r}\right.\right. \\
\left.\left.+e_{1}^{\prime} v_{1}^{\prime}+\cdots+e_{s}^{\prime} v_{s}^{\prime}\right)\right) .
\end{array}
$$

These are evident by inspection.

The multipliers $e_{2} e_{3} \cdots e_{s}, e_{1}^{\prime} e_{2}^{\prime} \cdots e_{s}^{\prime}$ on the right of (53), (54) are called the respective signs of the particular general terms of the summations, and also of the respective functions on the left. In (52) we define the sign to be +1 . The trace of each function on the left of (52)-(54), for all values of $x$, is that part of the argument of the $f$ on the right which contains only $u_{j}(j=1, \cdots, r)$ and $v_{k}(k=1, \cdots, s)$, or one of these sets if the other is absent. In particular, then, $\varphi_{n}\left(U_{r}\right), \psi_{n}\left(V_{s}\right), \chi_{n}\left(U_{r} ; V_{s}^{\prime}\right)$ have the same respective traces and signs as the functions in (52)-(54). We shall denote the trace of $F$ by $T(F)$, and its sign by $S(F)$. Clearly $S\left(F_{1} F_{2}\right)=S\left(F_{1}\right) S\left(F_{2}\right)$. When several functions $F, F_{1}, F_{2}, \cdots$ involving units occur, the units are treated as umbrae, so that, in particular, units arising in any formula from different functions are to be denoted by different systems of letters, thus $e_{j}, e_{k}{ }^{\prime}$, $e_{l}^{\prime \prime}, \ldots$. The necessity for this convention will be obvious from (55).

Let now each of $\lambda, \mu, \cdots, \nu$ denote a definite one of $\varphi, \psi, \chi$ for any systems $U_{a}, U_{b}, \cdots, V_{r}, V_{s}, \cdots$ of arguments $u_{j}, u_{k}{ }^{\prime}, \cdots, v_{l}, v_{m}{ }^{\prime}, \cdots$ $(j, k, \cdots, l, m, \cdots=1,2, \cdots)$. Then we have the fundamental theorem

$$
f(x+\lambda+\mu+\cdots+\nu)=\Sigma_{\sigma} S(\lambda \mu \cdots \nu) f(x+T(\lambda)+T(\mu)+\cdots+T(\nu)) .
$$


To illustrate the meaning of this for a particular case, let

$$
\lambda_{n} \equiv \varphi_{n}\left(U_{r}\right), \quad \mu_{n} \equiv \psi_{n}\left(V_{s}\right), \quad \nu_{n} \equiv \chi_{n}\left(U_{p^{\prime}} ; V_{\imath}^{\prime}\right) .
$$

Then, remembering the convention about units, we write the traces

$$
\begin{aligned}
& T(\lambda) \equiv u_{1}+e_{2} u_{2}+\cdots+e_{r} u_{r}, \quad T(\mu) \equiv v_{1}+e_{2}^{\prime} v_{2}+\cdots+e_{s}^{\prime} v_{s}, \\
& T(\nu) \equiv u_{1}^{\prime}+e_{2}^{\prime \prime} u_{2}^{\prime}+\cdots+e_{p}^{\prime \prime} u_{p}^{\prime}+e_{1}^{\prime \prime \prime} v_{1}^{\prime}+e_{2}^{\prime \prime \prime} v_{2}^{\prime}+\cdots+e_{t}^{\prime \prime \prime} v_{t}^{\prime \prime \prime},
\end{aligned}
$$

getting for $S(\lambda), S(\mu), S(\nu)$ respectively the values

$$
1, e_{2}{ }^{\prime} e_{3}^{\prime} \cdot \cdots e_{8}^{\prime}, e_{1}^{\prime \prime \prime} e_{2}^{\prime \prime \prime} \ldots e_{t}^{\prime \prime \prime} \text {. }
$$

Hence the sign is here $e_{2}{ }^{\prime} e_{3}{ }^{\prime} \cdots e_{8}^{\prime} e_{1}^{\prime \prime \prime} e_{2}^{\prime \prime \prime} \cdots e_{t}^{\prime \prime \prime}$, and the signature is $\Sigma_{2}^{\prime} e_{j}+\Sigma_{2}^{s} e_{k}^{\prime}+\Sigma_{2}^{p} e_{l}^{\prime \prime}+\Sigma_{1}^{i} e_{m}^{\prime \prime \prime}$, so that $\Sigma_{\sigma}$ consists of $2^{r+s+s+p-3}$ terms.

The concept of umbral equality applied to (55) leads to the simplest and most powerful process of the whole algebra. Let $\xi, \zeta$ be umbrae for which

$$
\xi^{n} \equiv \xi_{n}=\zeta_{n} \equiv \zeta^{n} \quad(n=0,1,2, \cdots) .
$$

Then we say that the umbrae $\xi, \zeta$ are equal, $\xi=\zeta$, and evidently in any theorem an umbra may be replaced by any other which is equal to it. Hence from (55) we have

$$
\begin{gathered}
\tau=\lambda+\mu+\cdots+\nu \\
. \supset . f(x+\tau)=\Sigma_{\sigma} S(\lambda \mu \cdots \nu) f(x+T(\lambda)+T(\mu)+\cdots+T(\nu)) .
\end{gathered}
$$

Although this is obvious it enables us in the applications to dispense with masses of algebraic computation and to reach in one step results for sequences of special functions which hitherto have been attained only by laborious reductions. It is evidently but a special case of the obvious implication

$$
\xi=\zeta \cdot \supset \cdot f(x+\xi)=f(x+\zeta) .
$$

Let next $f(x)$ be a power series* in $x$, or a function which can be expanded into such a series. Then, provided the series for $f(x), f(x+\lambda), \cdots$ are convergent for some $|x|>0$, all the results of this section remain valid for the new interpretation of $f(x)$.

If the series $f(x), f(x+\lambda), \cdots$ are divergent, there still is an extremely useful interpretation of all the formulas of this section. This method of

* When $f(x)$ is a Laurent expansion the algebra is also applicable with a few obvious restatements of theorems to include both positive and negative ranks of coefficients. A detailed example of the algebra in this case is given in the paper, cited in the introduction, on Spitzer's functions. 
interpretation was fully discussed in the paper on Euler algebra mentioned in the introduction. Briefly, the interpretation merely states the equality of the particular general coefficients in the series obtained by operations in a field from any given set of series, the addition, multiplication, division, subtraction of the field being defined in a specific way, for which see the paper cited. The equality in question can be independently established by means of only the finite processes which we have already discussed. The advantage, however, of using infinite processes is indisputable, and consists in the automatic presentation of valuable transformations of finite formulas which otherwise would probably be overlooked.

Finally it is clear from the way in which they were derived that all results in this section remain true when $x$, the constants $a, b$, and all arguments occurring in $f$ are interpreted as umbrae. In such interpretations the usual precautions regarding umbral derivatives must be observed. Thus $D_{\lambda} \sin \lambda x=\lambda \cos \lambda x$, and the umbral multiplication $\lambda \times \cos \lambda x$, must be performed before degradation of exponents (passage to ordinaries).

8. The connection of $\S \S 2-7$ with sequences is made as follows: Let $u_{n}, v_{n}, \cdots, w_{n}(n=0,1,2, \cdots)$ be any sequences of numbers (in which case $n$ is the only independent variable) or functions (when $n$ is not the only variable), and let each of $\lambda, \mu, \cdots, \nu$ be a definite one of $\varphi, \psi, \chi$ with umbral arguments chosen from among $u, v, \cdots, w$. Then $\lambda_{n}, \mu_{n}, \cdots, \nu_{n}$ $(n=0,1,2, \cdots)$ are new sequences of numbers or functions, and the algebra gives the means of rapidly determining their interrelations with the original set of sequences.

The reason for investigating a set of sequences based upon $\varphi, \psi, \chi$ rather than upon some other functions is pragmatic. This is established by actually applying the method to numerous well known functions and to the discovery of others with equally interesting properties. Some of the possibilities were mentioned in the introduction. And although as a matter of logic success in application is the only possible proof of a pragmatic proposition, we can yet see an obvious reason why the algebra does work. Define $\sum(-1)^{n} u_{2 n+1} t^{2 n+1} /(2 n+1) !, \sum(-1)^{n} u_{2 n} t^{2 n} /(2 n) !$, where $t$ is a parameter, to be the respective generators of the sequences $u_{2 n+1}, u_{2 n}(n=$ $0,1,2, \cdots)$, and for simplicity suppose that for some $|t|>0$ all generators discussed are absolutely convergent. Then clearly we shall not have exhausted the mutual relationships of the sequences $u_{n}, v_{n}, \cdots, w_{n}(n=$ $0,1,2, \cdots)$ until we have fully investigated the field generated by their generators (all the elements of this field being themselves generators). In this field the functions $\varphi, \psi, \chi$ with umbral arguments $u, v, \cdots, w$, or $\varphi, \psi, \chi$ functions of these, and so on, are precisely those functions which 
are generated by multiplication and division from the original generators (the basis of the field). As shown in the paper on Euler algebra there is no necessity to consider the convergence of the elements of this field, so that in all cases the derived elements are significant. Or, as pointed out in the introduction, convergence can be ignored otherwise by operating in a certain algebra with an infinite basis.

Occasionally with well known functions $u_{n}, v_{n}, \cdots, w_{n}$ it is more advantageous to discuss not these but $p_{n} u_{n}, q_{n} v_{n}, \cdots, r_{n} w_{n}$, where $p_{n}, q_{n}, \cdots, r_{n}$ are functions of $n$ alone. Accordingly we first work out the theory for the modified functions, translating only the final formulas, if desired, into terms of $u_{n}, v_{n}, \cdots, w_{n}$. This is the case for example with Spitzer's functions, where the appropriate multiplier is $(-1)^{n}$. Or again $u_{n}+p_{n}, v_{n}+q_{n}, \cdots$ may be more amenable than $u_{n}, v_{n}, \cdots$. This is so for the usual Bernoullian functions, which we slightly modify. Our functions can readily be expressed in terms of others in the literature by means of $\$ 14$.

Part II. The functions $\beta, \gamma, \eta, \rho$

9. Only sufficient need be given to illustrate the application of some of the chief processes of Part I to the Bernoullian functions $\beta_{n}(u), \gamma_{n}(u)$ and the Eulerian $\eta_{n}(u), \rho_{n}(u)$, and to suggest that a systematic treatment of $\beta, \gamma, \eta, \rho$ from this point of view should prove profitable.

The even suffix notation is used for the numbers $B, G, E, R$ of Bernoulli, Genocchi, Euler and Lucas, so that with the exceptions $B_{1}=-1 / 2, G_{1}=1$, all the numbers of odd ranks vanish, and the first seven values are

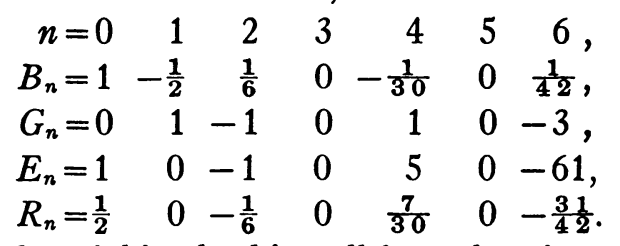

These will be found useful in checking all formulas given later. The symbolic generators, equivalent to definitions, are

$$
\begin{array}{rlrl}
x \cot x & =\cos 2 B x, & -x & =\sin 2 B x, \\
2 x \tan x & =\cos 2 G x, & 2 x & =\sin 2 G x, \\
\sec x & =\cos E x, & 0 & =\sin E x, \\
x \csc x & =2 \cos R x, \quad 0 & =\sin R x .
\end{array}
$$

The first column contains the definitions of Lucas, the second is a new and essential detail in the efficient application of the algebra.

In illustration now of the remarks in $\$ 8$ we may ask what are the relations of the sequences $B_{n}, G_{n}, E_{n}, R_{n}$ to any other sequences whatever, 
$u_{n}, v_{n}, w_{n}, \cdots(n=0,1,2, \cdots)$ ? Confining ourselves to the algebra of order 2 we may completely answer the question by combining the symbolic generators $\operatorname{soc} u x$, soc $v x$, soc $w x, \cdots$, where "soc" is an abbreviation for "sin or cos," with the definitions (58), and this immediately reduces the investigation to that of functions $\varphi, \psi, \chi$ with umbral arguments chosen from among $2 B, 2 G, E, R, u, v, w, \cdots$, and to $\varphi, \psi, \chi$ functions of these functions. Thus the solution is contained in the algebra constructed in Part I.

The simplest possibility is that of a single umbra $u$, and the special case of this in which $u_{n}=1(n=0,1,2, \cdots)$, so that the sequence is $1,1,1, \cdots$, gives the theory of the relations between the numbers $B, G, E, R$. The next simplest specialization for a single umbra $u$ is that in which $u_{n} \equiv u^{n}, u$ an ordinary, giving the theory of relations between the ordinary Bernoullian and Eulerian functions. Unless otherwise noted we shall take $u$ ordinary.

We can choose as fundamental any functions $\lambda(u, A)$ in which $\lambda$ is $\varphi$ or $\psi$ and $A$ is $2 B, 2 G, E$ or $R$. Taking $\lambda=\varphi$ we define the four fundamental functions

$$
\beta_{n}(u)=\varphi_{n}(u, 2 B), \gamma_{n}(u)=\varphi_{n}(u, 2 G), \eta_{n}(u)=\rho_{n}(u, E), \rho_{n}(u)=\varphi_{n}(u, R),
$$

whose symbolic generating identities are therefore (cf. (58))

$$
\begin{aligned}
& \operatorname{soc} \beta(u) x=2 x \cot x \operatorname{soc} u x \equiv 2 \cos 2 B x \operatorname{soc} u x, \\
& \operatorname{soc} \gamma(u) x=4 x \tan x \operatorname{soc} u x \equiv 2 \cos 2 G x \operatorname{soc} u x, \\
& \operatorname{soc} \eta(u) x=2 \sec x \operatorname{soc} u x \equiv 2 \cos E x \operatorname{soc} u x, \\
& \operatorname{soc} \rho(u) x=x \csc x \operatorname{soc} u x \equiv 2 \cos R x \operatorname{soc} u x,
\end{aligned}
$$

and whose non-symbolic forms are

$$
\begin{aligned}
& \beta_{n}(u)=2 \sum_{r=0} 2^{2 r}\left(\begin{array}{c}
n \\
2 r
\end{array}\right) B_{2 r} u^{n-2 r}, \\
& \eta_{n}(u)=2 \sum_{r=0}\left(\begin{array}{c}
n \\
2 r
\end{array}\right) E_{2 r} u^{n-2 r},
\end{aligned}
$$

with $\gamma_{n}(u)$ written down from $\beta_{n}(u)$ by replacing $B$ by $G$, and $\rho_{n}(u)$ from $\eta_{n}(u)$ by replacing $E$ by $R$. We have also from (4), (59)-(62) the equivalent identities

$$
\begin{aligned}
& \sin \beta(u) x=\sin \chi(2 B ; u) x, \quad \cos \beta(u) x=\cos \varphi(2 B, u) x, \\
& \sin \gamma(u) x=\sin \chi(2 G ; u) x, \quad \cos \gamma(u) x=\cos \varphi(2 G, u) x,
\end{aligned}
$$




$$
\begin{array}{ll}
\sin \eta(u) x=\sin \chi(E ; u) x, & \cos \eta(u) x=\cos \varphi(E, u) x, \\
\sin \rho(u) x=\sin \chi(R ; u) x, & \cos \rho(u) x=\cos \varphi(R, u) x .
\end{array}
$$

These with (4) give the best set of definitions for the application of Part I to $\beta, \gamma, \eta, \rho$. A complete discussion of these functions presupposes the like for the numbers $B, G, E, R$, viz., the special case already mentioned. Here it will be sufficient to derive such relations as are needed for purposes of illustration.

We restricted $u$ to be an ordinary. But all that follows holds also when $u$ is an umbra, and similarly for the variables $x, v, w, \cdots$. For definiteness, however, in reading formulas, $x, u, v, w, \cdots$ may be interpreted as their special cases, ordinaries. The more general interpretation, however, is a necessity, as will be seen, in the steps intermediate between an ordinary formula and its transforms.

10. Instead of the functions $\lambda_{n}(u)(\lambda=\beta, \gamma, \eta, \rho)$ of argument $u$ we can treat in precisely the same way the functions $\lambda_{n}(g(u))$ having as arguments any function $g(u)$ of $u$, by replacing in (59)-(66) $u$ by $g(u)$. For example, if $u$ is a positive integer, the functions in which $g(u)=2 u$ or $2 u-1$ are among those most frequently appearing in connection with sums of like powers of the integers $1,2, \cdots, n$ and similar problems. For the same $g(u)$, $u$ complex, the $\lambda_{n}(g(u))$ are but slightly different from the Bernoullian and Eulerian functions as most commonly defined. It is convenient in such cases to define new Bernoullian ard Eulerian functions $\Lambda_{n}(u)$ of argument $u$; thus

$$
\Lambda_{n}(u) \equiv \lambda_{n}(g(u)), \quad(\Lambda, \lambda) \equiv(B, \beta), \quad(G, \gamma), \quad(E, \eta), \quad(R, \rho) .
$$

There need be no confusion between $B_{n}(u)$ and $B_{n}$ if the former never is written without the argument $u$, and so for the rest. The definitions now become

$$
\operatorname{soc} B(u) x=\operatorname{soc} \beta(g(u)) x=2 \cos 2 B x \operatorname{soc} g(u) x,
$$

and so for the others, all being given by

$$
\operatorname{soc} \Lambda(u) x=\operatorname{soc} \lambda(g(u)) x .
$$

We now prove a general theorem regarding transformations of $\Lambda_{n}(u)$.

Jacobi proved the important result for the common Bernoullian functions, in which $g(u)=2 u-1$, that they are invariant to within sign when $u$ is replaced by $1-u$, and Raabe did the like for the common Eulerian functions. Generalizing this to any $g(u)$ we see from (67) that the necessary 
and sufficient condition that $\Lambda_{n}(u)(n=0,1,2, \cdots)$ be invariant to within sign when $u$ is replaced by $h(u)$ is that $g(h(u))= \pm g(u)$; in particular

$$
\begin{gathered}
g(h(u))=g(u) . \supset . \Lambda_{n}(h(u))=\Lambda_{n}(u), \\
g(h(u))=-g(u) . \supset . \Lambda_{n}(h(u))=(-1)^{n} \Lambda_{n}(u) .
\end{gathered}
$$

Thus the automorphic transformations of $\Lambda_{n}(u)(n=0,1,2, \cdots)$ are identical with those of $g(u)$.

As an example, excluding the trivial case $q a-p b=0$, we have

$$
\Lambda\left(u^{\prime}\right) \equiv \lambda_{n}\left(\frac{a u^{\prime}+b}{p u^{\prime}+q}\right)=(-1)^{n} \lambda_{n}\left(\frac{a u+b}{p u+q}\right) \equiv(-1)^{n} \Lambda_{n}(u),
$$

where

$$
u^{\prime}=-\frac{(q a+p b) u+2 q b}{2 p a u+(q a+p b)}
$$

and hence for $p=0, q=1, \lambda_{n}(a u+b)$ is transformed into $(-1)^{n} \lambda_{n}(a u+b)$ by the substitution $\{u,-u-2 b / a\}$, so that in the further special case $(a, b)=(2,-1), \lambda_{n}(2 u-1)$ is transformed into $(-1)^{n} \lambda_{n}(2 u-1)$ by $\{u, 1-u\}$, giving the theorems of Jacobi and Raabe.

11. From $\beta_{n}(u)=\varphi_{n}(u, 2 B)$ we find, by (10), the last $\chi_{2 n}$ in the same section, and (17),

$$
\beta_{n}(u+v)=(u+\beta(v))^{n}=(v+\beta(u))^{n},
$$

and hence, what is otherwise evident from the definition of $\beta_{n}(u)$, the addition theorem

$$
f(x+\beta(u+v))=f(x+u+\beta(v))=f(x+v+\beta(u)) .
$$

There are several more interesting types, of which one follows. From (59) on replacing $u$ by $u+v$ we get

$$
2 x \operatorname{soc} \beta(u+v) x=\tan x \operatorname{soc}(\beta(u)+\beta(v)) x,
$$

and hence from (60),

$$
8 x^{2} \operatorname{soc} \beta(u+v) x=\operatorname{soc} \gamma(\beta(u)+\beta(v)) x,
$$

so that finally by $(47),(49)$ we have

$$
f(x+\gamma(\beta(u)+\beta(v)))=-8 f^{\prime \prime}(x+\beta(u+v)) .
$$


In the same way

$$
\begin{aligned}
& f(x+\beta(\gamma(u)+\gamma(v)))=-8 f^{\prime \prime}(x+\gamma(u+v)), \\
& f(x+\eta(u)+\eta(v)+1)+f(x+\eta(u)+\eta(v)-1)=f(x+\eta(u+v)), \\
& f(x+\rho(u)+\rho(v)+1)-f(x+\rho(u)+\rho(v)-1)=2 f^{\prime}(x+\rho(u+v)) .
\end{aligned}
$$

12. The use of the umbral equalities and substitutions is illustrated by the following example. Suppose we wish to develop a function of $\beta(u)+c \beta(v)$, where $c=1$ or -1 , in powers of $u+c v$. We have $\beta(u)=\varphi(u$, $2 B), \beta(v)=\varphi\left(v, 2 B^{\prime}\right)$, where the identical umbrae $B, B^{\prime}$ are distinguished in the notation, as they are to occur in a single formula. Hence

$$
f(x+\beta(u)+c \beta(v))=f\left(x+\varphi(u, 2 B)+c \varphi\left(v, 2 B^{\prime}\right)\right),
$$

and therefore

$$
f(x+\beta(u)+c \beta(v))=\Sigma_{\sigma} f\left(x+2 e_{1} B+2 e_{2} B^{\prime}+u+c v\right) .
$$

To illustrate the same principle we shall obtain this in a more suggestive and essentially simpler manner. From $\beta(u)=\varphi(u, 2 B)$ we have by (57), (56)

$$
f(x+\beta(u))=f(x+\varphi(u, 2 B))=\Sigma_{\sigma} f\left(x+u+2 e_{1} B\right),
$$

valid for $x$ umbral. Take $x=x^{\prime}+c \beta(v)$, and write $x^{\prime}+u+2 e_{1} B \equiv x^{\prime \prime}$. Then

$$
f\left(x^{\prime}+\beta(u)+c \beta(v)\right)=f\left(x^{\prime \prime}+\varphi(v, 2 B)\right)=\Sigma_{\sigma} f\left(x^{\prime \prime}+v+2 e_{2} B\right) ;
$$

whence, on restoring the value of $x^{\prime \prime}$ and replacing $x^{\prime}$ by $x$, we have (73).

A relation involving functions $f$ of $\beta, \gamma, \eta, \rho$ is called of order $r$ provided $r$ is the greatest number of equal or distinct umbrae chosen from those occurring in the argument of any $f$. Thus (73) is of order 2. A complete system of relations of order $r$ may be written out similarly to the above. By means of the umbral equalities between the numbers $B, G, E, R$ the relations can be transformed systematically in many ways. For example, from the reciprocal symmetry between $\beta, B$ and $\gamma, G$, evident in the definitions of $\beta, \gamma$, we can write duwn from (73)

$$
f(x+\beta(u)+c \gamma(v))=\Sigma_{\sigma} f\left(x+2 e_{1} B+2 e_{2} G+u+c v\right) .
$$

Now, if we had reproduced here the preliminary algebra of $B, G, E, R$ necessary for a complete discussion of $\beta, \gamma, \eta, \rho$, we should have seen immediately that the right of (74) is at once reducible, on account of the 
presence of $B, G$ together in a linear combination. As it is, the first pair in (58) serve the same purpose, giving by multiplication

$$
\cos \varphi(2 B, 2 G) x=4 x^{2}=\cos \psi(2 B, 2 G),
$$

and hence from (49) with $\lambda=0, r=1$,

$$
\begin{aligned}
& -8 f^{\prime \prime}(x)=f(x+\varphi(2 B, 2 G))+f(x-\varphi(2 B, 2 G)), \\
& -8 f^{\prime \prime}(x)=f(x+\psi(2 B, 2 G))+f(x-\psi(2 B, 2 G)) .
\end{aligned}
$$

From these by subtraction we get

$$
f(x+2 B-2 G)+f(x-2 B+2 G)=0,
$$

and hence, combining this with the result of addition,

$$
-8 f^{\prime \prime}(x)=\Sigma_{\sigma} f\left(x+2 e_{1} B+2 e_{2} G\right) \text {. }
$$

Apply this to the right of (74). Then

$$
f(x+\beta(u)+c \gamma(v))=-8 f^{\prime \prime}(x+u+c v) .
$$

In a complete exposition the derivation of (76) would be still shorter, for we should then have available all theorems such as (75) on the numbers $B, G, E, R$. By repetition of (76) we find a curious result. Replace $u$ by $\beta(u)$, $v$ by $\gamma(v)$, repeat the process $r-1$ times, using (76) each time to reduce the new right hand member to a form free from $\beta, \gamma$. Write $\beta_{n}(\beta(\cdots \beta(u)$ $\cdots))(r$ symbols $\beta) \equiv \beta_{n}{ }^{[r]}(u)$, and similarly for $\gamma$. Then

$$
f\left(x+\beta^{[r]}(u)+c \gamma^{[r]}(v)\right)=(-8)^{r} f^{\{r]}(x+u+c v) .
$$

In the same way, or as noted in a moment, we see the following interesting reciprocities between pairs of $\beta, \gamma, \eta, \rho(c= \pm 1)$ :

$$
\begin{aligned}
& f(x+\beta(\gamma(u)))=f(x+\gamma(\beta(u)))=-8 f^{\prime \prime}(x+u), \\
& f(x+\beta(\eta(u)))=f(x+\eta(\beta(u)))=4 f(x+\rho(u)), \\
& f(x+c \beta(\rho(u))+1)-f(x+c \beta(\rho(u))-1)=2 f^{\prime}(x+c \beta(u)),
\end{aligned}
$$

and the like with $\beta, \rho$ interchanged only on the left;

$$
f(x+\gamma(\eta(u))+1)+f(x+\gamma(\eta(u))-1)=4 f(x+\nu(u)),
$$

and the like with $\gamma, \eta$ interchanged only on the left;

$$
\begin{aligned}
& f(x+\gamma(\rho(u)))=f(x+\rho(\gamma(u)))=f(x+\eta(u)), \\
& f\left(x+\frac{1}{2} \eta(\rho(2 u))\right)=f\left(x+\frac{1}{2} \rho(\eta(2 u))\right)=f(x+\rho(u)) .
\end{aligned}
$$


The alternative derivation is from (59)-(62). Thus, for example, (59), (60) give at once

$$
\operatorname{soc} \beta(\eta(u)) x=\operatorname{soc} \eta(\beta(u)) x=4 \operatorname{soc} \rho(u) x,
$$

and therefore (79).

Many of the theorems of the types obtainable by the methods of this section immediately suggest investigations worth detailed development. For instance the presence of the identical umbrae $B, B^{\prime}$ in (73) directs us to introduce into the theory of the Bernoullian and allied functions the Bernoullian and allied numbers of higher order which were first defined by Lucas. Thus the right of (73) need involve only the single umbra $B^{(2)}$ of the Bernoullian numbers of the second order. This in turn suggests that we generalize the whole theory of the Bernoullian and Eulerian functions by introducing such functions of order $r \geqq 1$, and so obtain the theory of the ordinary Bernoullian and Eulerian functions by taking $r=1$ in the results. The general theory is as readily developed by means of the algebra in Part I as is its special case $r=1$.

13. We take space merely to indicate three of the far reaching applications of relations such as those in the preceding section. First, an indefinite number of curtate relations between functions $\beta, \gamma, \eta, \rho$ are obtainable by taking for $f(x)$ the product of any number of polynomials in $x$ of equal or unequal degrees.

Second, the relations between functions $\beta, \gamma, \eta, \rho$ whose ranks are the successive numbers of any arithmetic progression with first term and common difference positive integers are found uniformly as follows. Simson's well known method for summing the series obtained by selecting from any given series every $m$ th term leads at once, through the functions of Olivier and a corresponding generalization of the symmetric functions of Kronecker, to the algebra of order $m$ (cf. $\$ 1$ ), when for the given series we take the expansion of an exponential, circular or hyperbolic function. Multiplying the $n$th term of the new series by $u_{n}$ or $v_{n}$, or $w_{n}, \cdots$, we obtain precisely as in Part I a set of umbral generators from which we construct as there the algebra of these generalized umbral series and cosines. Clearly if $u_{n}=v_{n}=w_{n} \cdots=1(n=0,1,2, \cdots)$, the result of multiplying any series by a so specialized umbral sine or cosine of the new type selects from the series all terms whose ranks are in arithmetic progression with common difference $m$. For other sequences $u_{n}, v_{n}, w_{n}, \cdots$, the selected series are a generalization of these, and lead to an extension in a wholly new direction of the Bernoullian and Eulerian functions. The entire theory of the relations between the coefficients of the selected series is isomorphic to that 
of the indicated generalization of Olivier's functions, and is obtained with great ease by means of the algebra of order $m$. The case $m=2$ developed in Part $I$ is a complete guide to $m>2$.

Third, the application of all that precedes to the special case in which each of the variables $u_{i}, v_{k}, \cdots$ is an integer is of importance in the theory of numbers, as it leads to many congruences for the numbers $B, G, E, R$.

For rational arguments the functions $\beta, \gamma, \eta, \rho$ yield the theory of sums of like powers of integers in arithmetic progression, or such sums with periodic successions of \pm signs. Thus it is easily seen that if $u, a, b$ are positive integers, and

$$
b \sigma_{n}(u) \equiv 2 \sum_{j=0}^{u}(a+b j)^{n}, \quad b v \equiv 2 a+(2 u+1) b, \quad b w \equiv 2 a-b,
$$

then (from the expansion of $\left.\sum_{0}^{u} 2 x p(a+b j) x\right)$,

$$
f(x+\rho(v))-f(x+\rho(w))=2 f^{\prime}(x+\sigma(u)) .
$$

14. For comparison with other Bernoullian functions in the literature we add the exponential definitions of the generators. These are found from (59)-(62) or from (63)-(66) by writing down the values of

$$
\begin{gathered}
\cos \{\lambda(u) x / i\}+i \sin \{\lambda(u) x / i\} \quad(\lambda=\beta, \gamma, \eta, \rho): \\
e^{\beta(u) x}=2 x e^{u x}\left(\frac{e^{2 x}+1}{e^{2 x}-1}\right), \quad e^{\gamma(u) x}=-4 x e^{u x}\left(\frac{e^{2 x}-1}{e^{2 x}+1}\right), \\
e^{\eta(u) x}=4 e^{u x}\left(\frac{e^{x}}{e^{2 x}+1}\right), \quad e^{\rho(u) x}=2 x e^{u x}\left(\frac{e^{x}}{e^{2 x}-1}\right) .
\end{gathered}
$$

UNIVERSITY OF WASHINGTON,

Seattle, Wash. 